\title{
Prevalence of myocardial viability assessed by single photon emission computed tomography in patients with chronic ischaemic left ventricular dysfunction
}

\author{
A F L Schinkel, J J Bax, F B Sozzi, E Boersma, R Valkema, A Elhendy, J R T C Roelandt, \\ D Poldermans
}

Heart 2002;88:125-130

\begin{abstract}
Objective: To assess the prevalence of myocardial viability by technetium-99m (Tc-99m)-tetrofosmin/ fluorine-18-fluorodeoxyglucose (FDG) single photon emission computed tomography (SPECT) in patients with ischaemic cardiomyopathy.

Design: A retrospective observational study.

Setting: Thoraxcenter Rotterdam (a tertiary referral centre).

Patients: 104 patients with chronic coronary artery disease and severely depressed left ventricular function presenting with heart failure symptoms. Main outcome measures: Prevalence of myocardial viability as evaluated by Tc-99m-tetrofosmin/ FDG SPECT imaging. Two strategies for assessing viability in dysfunctional myocardium were used: perfusion imaging alone, and the combination of perfusion and metabolic imaging.

Results: On perfusion imaging alone, 56 patients (54\%) had a significant amount of viable myocardium, whereas 48 patients $(46 \%)$ did not. Among the 48 patients with no significant viability by perfusion imaging alone, seven additional patients (15\%) had significantly viable myocardium on combined perfusion and metabolic imaging. Thus with a combination of perfusion and metabolic imaging, 63 patients $(61 \%)$ had viable myocardium and $41(39 \%)$ did not.

Conclusions: On the basis of the presence of viable dysfunctional myocardium, $61 \%$ of patients with chronic coronary artery disease and depressed left ventricular ejection fraction presenting with heart failure symptoms may be considered for coronary revascularisation. The combination of perfusion and metabolic imaging identified more patients with significant viability than myocardial perfusion imaging alone.
\end{abstract}

See end of article for authors' affiliations

C hronic coronary artery disease is the most important cause of left ventricular dysfunction leading to heart failure. When this occurs it has an extremely poor prognosis. ${ }^{12}$ In nearly $70 \%$ of the patients in 13 major heart failure trials, coronary artery disease was the underlying cause of the heart failure. ${ }^{3}$ The management of these patients remains difficult, while both the incidence and the prevalence of chronic heart failure have been increasing in recent years. ${ }^{4}$ Medical treatment is still suboptimal in these patients, although significant improvements in survival have been achieved with angiotensin converting enzyme (ACE) inhibition, blockade of aldosterone receptors by spironolactone, and $\beta$ adrenergic blockade. ${ }^{5-7}$ Other therapeutic options are heart transplantation and coronary revascularisation. The possibilities of heart transplantation are limited by the availability of donor hearts, but coronary revascularisation could be an effective alternative. In a subset of patients with heart failure secondary to chronic coronary artery disease, revascularisation has been shown to improve left ventricular ejection fraction (LVEF), heart failure symptoms, and survival. ${ }^{8}$ However, revascularisation in such patients is also associated with increased periprocedural morbidity and mortality. ${ }^{89}$

The concept of myocardial viability was proposed to explain the improvement of LVEF and heart failure symptoms after revascularisation. ${ }^{10}{ }^{11}$ Dysfunctional but viable myocardium is likely to regain contractile function after coronary revascularisation. ${ }^{12}$ On the other hand, dysfunctional nonviable myocardium (scar tissue) clearly will not improve. Bonow estimated that between 25-40\% of patients with heart failure caused by ischaemic heart disease have the potential for functional improvement after revascularisation. ${ }^{13}$ Quantifi- cation of myocardial viability in patients with chronic coronary artery disease, depressed left ventricular function, and heart failure is at present limited, but this information is important as such patients may benefit from revascularisation. ${ }^{14}{ }^{15}$ Our aim in this study was therefore to evaluate myocardial viability in a consecutive series of 104 patients with chronic coronary artery disease and depressed LVEF presenting with heart failure symptoms.

\section{METHODS}

Patient population, study protocol

The study population consisted of 104 consecutive patients with ischaemic cardiomyopathy (chronic coronary artery disease as assessed by angiography and an LVEF of $\leqslant 35 \%$ ), who presented with heart failure as the predominant symptom and were referred for evaluation of myocardial viability. Patients with primary cardiomyopathy or concomitant significant valvar disease were not included.

All patients underwent resting echocardiography to identify dysfunctional myocardial tissue, and dual isotope simultaneous acquisition myocardial single photon emission computed tomography (SPECT), including Tc-99m tetrofosmin and fluorodeoxyglucose (FDG) to assess myocardial perfusion and

Abbreviations: ACE, angiotensin converting enzyme; FDG, fluorodeoxyglucose; LVEF, left ventricular ejection fraction; PET, positron emission tomography; ROC, receiver operating characteristic; SPECT, single photon emission computed tomography 
glucose utilisation, respectively. ${ }^{16}{ }^{17}$ LVEF was assessed by radionuclide ventriculography.

The local medical ethics committee approved the protocol and all patients gave informed consent.

\section{Assessment of contractile function}

A Hewlett-Packard Sonos-5500 imaging system (HewlettPackard Inc, Andover, Massachusetts, USA), equipped with a 1.8 MHz transducer using second harmonic imaging to optimise endocardial border visualisation, was used to record cross sectional echocardiograms. Four standard views (apical two and four chamber views, and parasternal short and long axis views) were stored on tape.

Off-line interpretation was undertaken using a computer system that digitised the taped images and displayed them in a cineloop format. Two experienced reviewers blinded to the SPECT data scored the digitised echocardiograms visually. In case of disagreement, a majority decision was achieved by a third reviewer. The left ventricle was divided according to the standard 16 segment model described by the American Society of Echocardiography ( six basal, six distal, and four apical segments). ${ }^{18}$ Regional wall motion and systolic wall thickening were scored using a five point grading scale: 1, normal (normal endocardial excursion and systolic wall thickening); 2, mildly hypokinetic (mildly reduced excursion and wall thickening); 3, severely hypokinetic ( severely reduced excursion and thickening); 4, akinetic (absent excursion and wall thickening); 5, dyskinetic (paradoxical systolic outward wall motion). Myocardial segments were considered normal if the regional wall motion was normal or mildly hypokinetic. Only segments with severe hypokinesia, akinesia, or dyskinesia were evaluated for myocardial viability.

\section{SPECT data acquisition}

After a light breakfast, the patients received an intravenous injection of Tc-99m-tetrofosmin (600 MBq) to evaluate resting perfusion. FDG imaging to evaluate glucose utilisation was done after the administration of acipimox (500 mg oral dose) in all patients. Acipimox enhances myocardial FDG uptake by reducing the plasma concentration of free fatty acids. Following acipimox administration, the patients received a low fat, carbohydrate rich meal. This small meal further enhances myocardial FDG uptake by stimulating endogenous insulin release. Several studies have shown excellent imaging using acipimox. ${ }^{19}$

Patients with diabetes mellitus were asked to continue their antidiabetic drug regimen. Plasma glucose concentrations were measured immediately before the study. In patients with a plasma glucose concentration of more than $8 \mathrm{mmol} / \mathrm{l}$, insulin was given intravenously to enhance myocardial FDG uptake. The plasma glucose concentration was then measured again and additional intravenous insulin given if necessary.

Sixty minutes after the meal, FDG ( $185 \mathrm{MBq}$ ) was injected, and after an a further 45 minutes to allow cardiac FDG uptake, dual isotope simultaneous acquisition SPECT was performed. Perfusion and metabolic imaging were both done at rest without stressors. Patients continued their normal cardiac drug treatment during the SPECT study.

A triple head gamma camera system (Picker Prism 3000XP, Cleveland, Ohio, USA) was used. The camera system was equipped with high energy $511 \mathrm{keV}$ collimators. The energies were centred on the $140 \mathrm{keV}$ photon peak of technetium-99m tetrofosmin with a 15\% window and on the $511 \mathrm{keV}$ photon peak of FDG with a $15 \%$ window. Data acquisition was done in the supine position, over $360^{\circ}\left(120\right.$ sectors of $\left.3^{\circ}\right)$. Total imaging time was 32 minutes. Data were stored in a $64 \times 64,16$ bit matrix.

\section{SPECT data reconstruction and analysis}

From the raw scintigraphic data, $6 \mathrm{~mm}$ thick ( 1 pixel) transaxial slices were reconstructed by filtered back projection using a
Table 1 Clinical characteristics of 104 patients undergoing dual isotope simultaneous acquisition single photon emission computed tomography

\begin{tabular}{|c|c|}
\hline Clinical features & $\begin{array}{l}\text { Number of } \\
\text { patients }\end{array}$ \\
\hline LVEF $\leqslant 20 \%$ & $23(22 \%)$ \\
\hline LVEF > $20 \%, \leqslant 30 \%$ & $54(52 \%)$ \\
\hline LVEF $>30 \%, \leqslant 35 \%$ & $27(26 \%)$ \\
\hline $\begin{array}{l}\text { Number of stenosed arteries } \\
\text { (mean (SD)) }\end{array}$ & $2.1(0.8)$ \\
\hline Previous MI & $97(93 \%)$ \\
\hline Previous CABG & $16(15 \%)$ \\
\hline Previous PTCA & $13(13 \%)$ \\
\hline Diabetes mellitus & 15 (14\%) \\
\hline \multicolumn{2}{|l|}{ Drug treatment } \\
\hline ACE inhibitors & $86(83 \%)$ \\
\hline$\beta$ Blockers & $53(51 \%)$ \\
\hline Calcium antagonists & $29(28 \%)$ \\
\hline Nitrates & $74(71 \%)$ \\
\hline Diuretics & $66(63 \%)$ \\
\hline \multicolumn{2}{|c|}{$\begin{array}{l}\text { Data are } n(\%) \text { unless specified. } \\
\text { ACE, angiotensin converting enzyme; CABG, } \\
\text { coronary artery bypass graft surgery; LVEF, left } \\
\text { ventricular ejection fraction; MI, myocardial } \\
\text { infarction; PTCA, percutaneous transluminal } \\
\text { coronary angioplasty. }\end{array}$} \\
\hline
\end{tabular}

Butterworth filter (cut off frequency at 0.17 cycle/pixel of order 3.5). Attenuation correction was not applied. Further reconstruction yielded standard short and long axis projections perpendicular to the heart axis. The Tc-99m-tetrofosmin and the FDG data were reconstructed simultaneously. This approach permits exact alignment of the perfusion and FDG images.

The perfusion and FDG short axis slices were adjusted to peak myocardial activity (100\%). The left ventricle was divided into 16 segments matching the echocardiographic segments. ${ }^{20}$ Both Tc-99m-tetrofosmin and FDG uptake defects were graded semiquantitatively on a four point scale: 0 , normal $(\geqslant 75 \%-$ $100 \%$ ); 1 , mildly reduced ( $\geqslant 50 \%-75 \% ; 2$, moderately reduced $(\geqslant 25 \%-50 \%) ; 3$, severely reduced or absent ( $\leqslant 25 \%$ uptake). Dysfunctional segments (identified by resting echocardiography) were subsequently evaluated for viability.

Viable myocardium was defined using two approaches: perfusion criteria alone, and a combination of perfusion and metabolic criteria. Using perfusion alone, myocardium with a To-99m-tetrofosmin uptake score of $\leqslant 1$ was considered viable, while myocardium with a score of $\geqslant 2$ was considered non-viable. Using the combination of perfusion and metabolism, myocardium with a Tc-99m-tetrofosmin uptake score of $\leqslant 1$ or with a reduction in Tc-99m-tetrofosmin uptake score that was more severe than the reduction in FDG activity by $\geqslant 1$ point (perfusion-metabolism mismatch pattern) was considered viable. Dysfunctional myocardium with a proportionate reduction in both Tc-99m-tetrofosmin and FDG uptake (perfusion-metabolism match pattern) was considered non-viable.

A patient with four or more dysfunctional but viable segments was considered to have a functionally significant amount of viable myocardium. This definition is based on previous work using receiver operating characteristic (ROC) curve analysis showing that improvement in LVEF after revascularisation can be anticipated when more than $25 \%$ of the left ventricle is viable (four or more segments in a 16 segment model). ${ }^{21}$

\section{Assessment of LVEF}

To assess LVEF at the time of the viability testing, we performed radionuclide ventriculography at rest in all patients. A small field of view gamma camera system (Orbiter, Siemens, Erlangen, Germany) was used, oriented in a $45^{\circ}$ left anterior oblique position with a $5-10^{\circ}$ caudal tilt. After injection of To-99m (740 MBq), radionuclide ventriculography was 


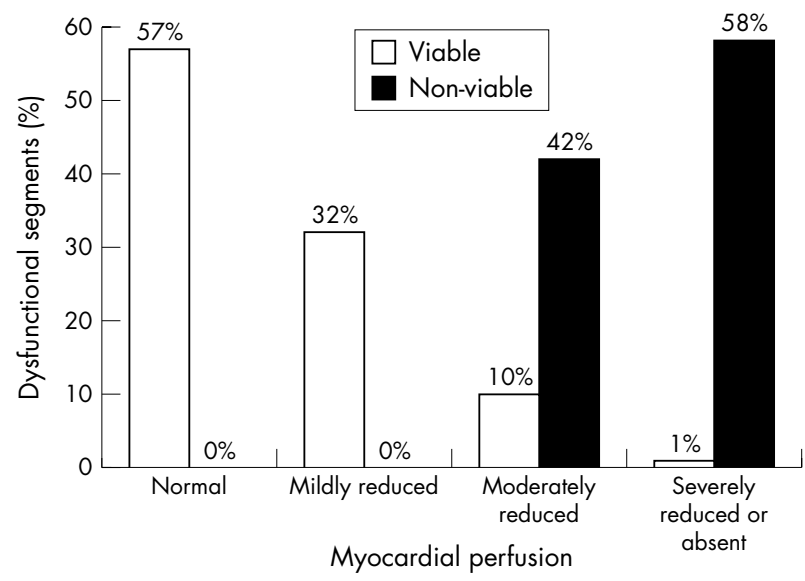

Figure 1 Myocardial perfusion in viable and non-viable tissue. In viable myocardium, perfusion was normal in $57 \%$ of dysfunctional segments, mildly reduced in $32 \%$, moderately reduced in $10 \%$, and severely reduced or absent in $1 \%$. Non-viable tissue had moderately reduced perfusion in $42 \%$ of dysfunctional segments and severely reduced or absent perfusion in 58\%.

done at rest with the patient in the supine position. The LVEF was calculated by standard methods (Odyssey VP, Picker, Cleveland, Ohio, USA).

\section{Statistical analysis}

All continuous data are expressed as mean (SD). Percentages are rounded. Comparisons were made using the Student $t$ test for unpaired samples. A probability value of $\mathrm{p}<0.05$ was considered significant.

\section{RESULTS}

\section{Patient characteristics}

Clinical characteristics of the 104 patients (87 men, 17 women; mean (SD) age, 60 (9) years) are summarised in table 1. All patients presented with symptoms of heart failure. The mean New York Heart Association (NYHA) functional class was 2.5 (0.9); 67 patients were in NYHA class III or IV. The majority (93\%) of the patients had suffered a previous myocardial infarct (all occurring more than one month before viability assessment). LVEF assessed by radionuclide ventriculography averaged 25 (7)\% (range 9-35\%). In 23 patients the LVEF was $20 \%$ or less, in 54 it was between $21 \%$ and $30 \%$, and in 27 it was more than $30 \%$.

\section{Segmental analysis}

\section{Contractile function}

Cross sectional echocardiographic analysis of regional left ventricular function was done in 1664 segments, of which 189 were normal and 297 mild hypokinetic. Of 1178 dysfunctional segments $(71 \%), 584$ showed severe hypokinesia, 578 akinesia, and 16 dyskinesia. The mean (SD) number of dysfunctional segments per patient was 11.3 (4.2).

Table 2 Comparison of segment viability in three groups divided by left ventricular ejection fraction

\begin{tabular}{|c|c|c|c|}
\hline $\begin{array}{l}\text { Characteristics of } \\
\text { segments }\end{array}$ & $\begin{array}{l}\text { LVEF } \leqslant 20 \% \\
\text { ( } 23 \text { patients) }\end{array}$ & $\begin{array}{l}\text { LVEF } 21-30 \% \\
\text { (54 patients) }\end{array}$ & $\begin{array}{l}\text { LVEF }>30 \% \\
\text { ( } 27 \text { patients) }\end{array}$ \\
\hline Dysfunctional & $14.1(2.0)$ & $10.9(4.5) \dagger$ & $9.7(3.9)$ \\
\hline Viable by Tc-99m & $6.4(3.7)$ & $4.6(3.8)^{*}$ & $3.8(3.2)$ \\
\hline $\begin{array}{l}\text { Viable by Tc-99m } \\
+ \text { FDG }\end{array}$ & $7.2(4.0)$ & $5.1(4.1)^{*}$ & $4.2(3.2)$ \\
\hline
\end{tabular}

${ }^{*} p<0.05 ; \dagger p<0.005 v \operatorname{LVEF} \leqslant 20 \%$

FDG, fluorodeoxyglucose; LVEF, left ventricular ejection fraction.
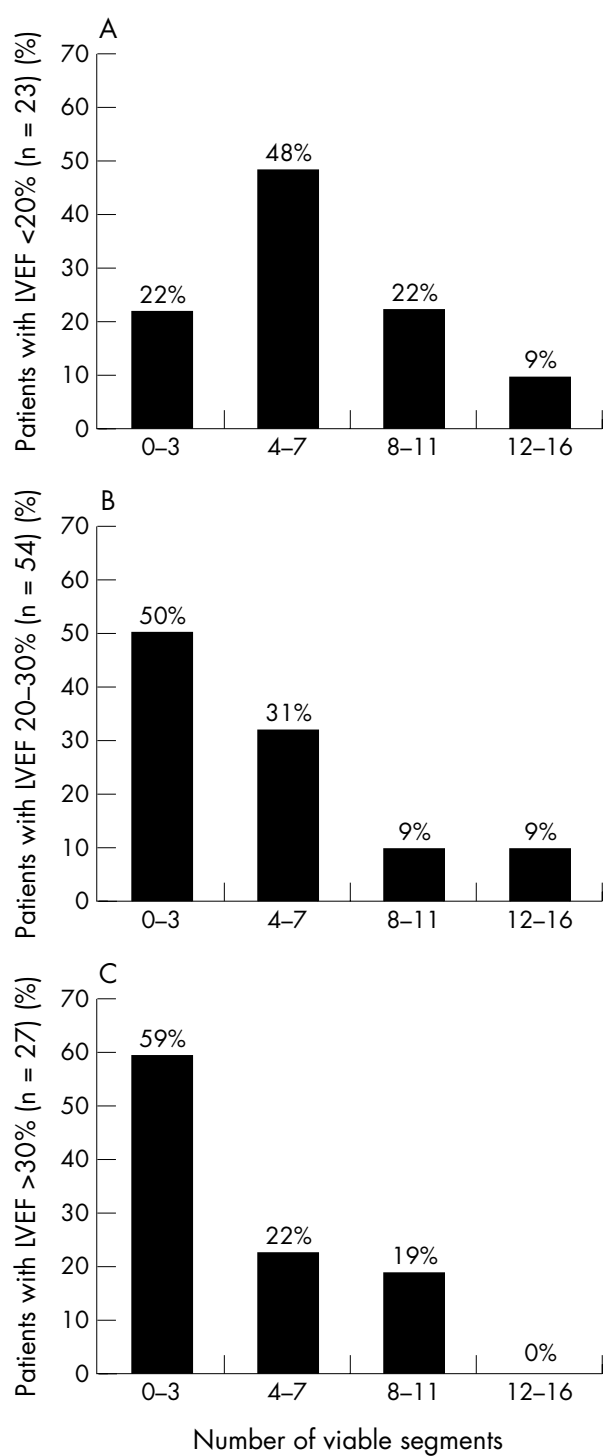

Figure 2 (A) Amount of viable myocardium indicated by the number of viable segments in 23 patients with a left ventricular ejection fraction (LVEF) $\leqslant 20 \%$. Using perfusion as the only criterion of viability, 18 patients $(78 \%$ ) had significant viability ( $\geqslant 4$ viable segments) and five (22\%) did not have significant viability.

(B) Amount of viable myocardium indicated by the number of viable segments in 54 patients with an LVEF $>20 \%$ but $\leqslant 30 \%$. Using perfusion as the only criterion of viability, 27 patients $(50 \%)$ had significant viability ( $\geqslant 4$ viable segments), and $27(50 \%)$ did not have significant viability. (C) Amount of viable myocardium indicated by the number of viable segments in 27 patients with an LVEF $>30 \%$ but $\leqslant 35 \%$. Using perfusion as the only criterion of viability, 11 patients $(41 \%)$ had significant viability ( $\geqslant 4$ viable segments) and $16(59 \%)$ did not have significant viability.

\section{Perfusion imaging alone}

Using myocardial perfusion as the only criterion of viability, 497 dysfunctional segments (42\%) were classified as viable. Three hundred and sixteen dysfunctional segments (27\%) had normal Tc-99m-tetrofosmin uptake, and 181 (15\%) had a mildly reduced uptake. The remaining 681 dysfunctional segments $(58 \%)$ had a Tc-99m tetrofosmin uptake score of 2 or 3 and were classified as non-viable.

Combined perfusion and metabolic imaging

Using perfusion imaging in conjunction with FDG SPECT, 558 of the dysfunctional myocardial segments (47\%) were classified as viable. Of these viable dysfunctional segments, 

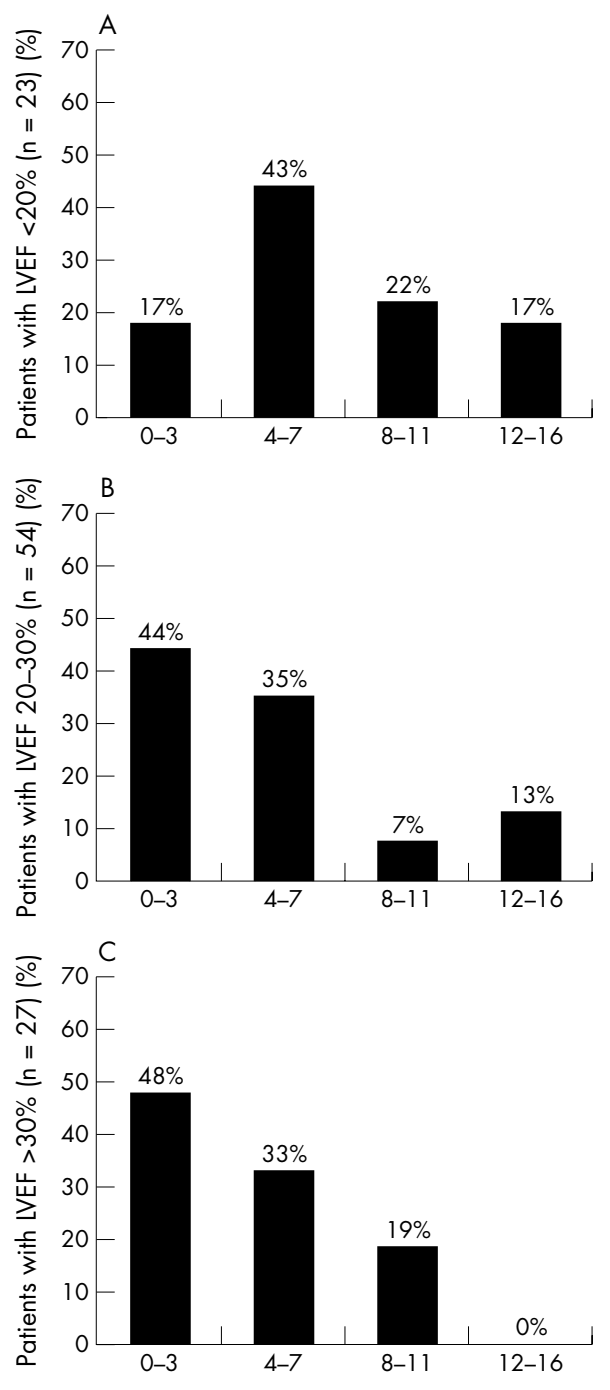

Number of viable segments

Figure 3 (A) Amount of viable myocardium indicated by the number of viable segments in 23 patients with a left ventricular ejection fraction (LVEF) $\leqslant 20 \%$. Using perfusion in combination with metabolic criteria for viability, 19 patients $(83 \%)$ had significant viability ( $\geqslant 4$ viable segments) and four (17\%) did not. (B) Amount of viable myocardium indicated by the number of viable segments in 54 patients with an LVEF $>20 \%$ but $\leqslant 30 \%$. Using perfusion in combination with metabolic criteria for viability, 30 patients $(56 \%)$ had significant viability ( $\geqslant 4$ viable segments) and $24(44 \%)$ did not. (C) Amount of viable myocardium indicated by the number of viable segments in 27 patients an LVEF $>30 \%$ but $\leqslant 35 \%$. Using perfusion in combination with metabolic criteria for viability, 14 patients $(52 \%)$ had significant viability ( $\geqslant 4$ viable segments) and $13(48 \%)$ did not.

497 (42\%) had normal or mildly reduced perfusion and 61 additional segments $(5 \%)$ had a perfusion-metabolism mismatch pattern. Conversely, 620 dysfunctional segments $(53 \%)$ were classified as non-viable (with a matching pattern of perfusion and metabolism). Figure 1 shows the myocardial perfusion in the dysfunctional segments that were classified as viable and non-viable by combined perfusion and metabolic imaging.

\section{Patient analysis}

\section{Contractile function}

Patients with an LVEF of $20 \%$ or less had more dysfunctional segments than those with an LVEF of $21-30 \%$ (14.1 (2.0) $v$ $10.9(4.5), \mathrm{p}<0.005)$ or those with an LVEF of more than $30 \%$ (14.1 (2.0) $v 9.7$ (3.9), $\mathrm{p}<0.0001)$. The number of dysfunc- tional segments was not significantly different between patients with an LVEF of more than $30 \%$ and those with an LVEF of 21-30\% (9.7 (3.9) $v 10.9(4.5) ; \mathrm{p}=0.2$ ) (table 2).

Perfusion imaging alone

All patients had on average 4.8 (3.7) (range 0-14) dysfunctional but viable segments. When myocardial perfusion was used as the only criterion of viability, 56 patients (54\%) had a significant amount of viable myocardium (on average 7.5 (2.9) segments) and 48 patients $(46 \%)$ did not have significant viability (1.6 (1.2) segments). Figure 2 shows the number of viable segments per patient with perfusion imaging alone for the three LVEF groups.

Combined perfusion and metabolic imaging

Among the 48 patients with no significant viability by perfusion imaging alone, seven additional patients (15\%) had viable myocardium when assessed using a combination of perfusion and metabolic imaging. All 56 patients with significant viability when assessed by perfusion alone also had significant viability with combined perfusion and metabolic imaging (on average, 8.2 (3.2) viable segments). Thus perfusion imaging in combination with metabolic imaging showed that 63 patients $(61 \%)$ had a significant amount of viable tissue (7.8 (3.2) segments). Forty one patients (39\%) did not have significant residual viable dysfunctional tissue (on average, 1.6 (1.2) viable segments). Overall, the patients had 5.4 (4.0) viable dysfunctional segments. Figure 3 shows the number of viable dysfunctional segments for patients in the three different LVEF categories.

\section{DISCUSSION \\ Viable myocardium}

Heart failure is becoming a major problem in clinical cardiology. In recent years the incidence of patients with heart failure has increased despite progress in prevention and treatment. ${ }^{4}$ Chronic heart failure is predominantly caused by coronary artery disease and has a poor prognosis. ${ }^{3}$ In a subset of patients with heart failure resulting from coronary artery disease, coronary revascularisation may improve left ventricular function, heart failure symptoms, and survival, ${ }^{8}{ }^{9}$ but the risks of revascularisation in such patients are substantial. Also, apart from the need to assess clinical variables such as target vessels and comorbid factors, it is important to evaluate myocardial viability as part of the complex selection process in candidates for revascularisation.

Previous estimates suggest that between $25-40 \%$ of the patients with heart failure caused by coronary artery disease may show useful functional improvement after revascularisation. ${ }^{13}$ In our hospital, patients with coronary artery disease, a depressed LVEF, and heart failure are always referred for assessment of myocardial viability. This approach allows improved risk stratification and results in optimal treatment for the individual patient. In the present study, we examined myocardial viability in a consecutive series of patients from our heart failure registry.

A few previous studies have examined myocardial viability on an individual patient basis. Using FDG positron emission tomography (PET), Pasquet and colleagues found that among 66 patients with severe left ventricular dysfunction, 28 (47\%) had an improvement in LVEF of at least 5\% after coronary revascularisation, while eight (14\%) had an improvement of more than $10 \%{ }^{22} \mathrm{Al}$-Mohammad and colleagues studied the prevalence of hibernating myocardium in 27 patients using FDG PET. ${ }^{14}$ Fourteen of these had significant areas of hibernating myocardium on PET. Auerbach and associates, also using FDG PET, studied 283 patients with ischaemic cardiomyopathy and found that 156 (55\%) had viable myocardium. ${ }^{15}$ In the present study, $54 \%$ of the patients showed significant viability when myocardial perfusion was 
used as the only index, while perfusion imaging in combination with metabolic imaging identified a further 7\%; thus $61 \%$ of the patients in all had a significant amount of viable tissue. These observations are in close agreement with those of Al-Mohammad and Auerbach using FDG PET. ${ }^{14} 15$

In the present study patients with an LVEF of $20 \%$ or less had more dysfunctional but viable segments than those with an LVEF of $21-30 \%$ or with an LVEF of more than $30 \%$. In line with our results, Fath-Ordoubadi and colleagues reported that in 47 patients with coronary artery disease and chronic left ventricular dysfunction, those with an LVEF of $30 \%$ or less had more dysfunctional but viable segments than those with an LVEF of more than $30 \% .{ }^{23}$ Moreover, after revascularisation the LVEF improved in patients with an initial LVEF of $30 \%$ or less, whereas it remained unchanged in those with an LVEF of more than $30 \%$. It seems that patients with the most severe left ventricular dysfunction benefit more from revascularisation than those with less severe dysfunction. These findings may have important clinical implications in relation to revascularisation, as severe left ventricular dysfunction has a very poor prognosis when treated medically. ${ }^{3}$

\section{How much viable tissue is required to be clinically relevant?}

In the present study a patient was considered to have a significant amount of viable myocardium if there were at least four or more dysfunctional viable segments in a 16 segment model. This definition was based on a previous study using ROC curve analysis showing that improvement in LVEF after revascularisation can be anticipated when $25 \%$ or more of the left ventricle is viable. ${ }^{21}$ Di Carli and colleagues reported that in patients with coronary artery disease and left ventricular dysfunction and with more than $5 \%$ viable tissue, the survival rate of revascularised patients was higher than in those who were treated medically $(88 \% \vee 50 \%){ }^{24}$ These data suggest that revascularisation in patients with $5-25 \%$ of viable myocardial tissue, while being unlikely to result in functional recovery, may still improve prognosis. Moreover, five prognostic FDG PET studies have reported a high event rate $(42 \%)$ in patients with viable tissue treated medically, ${ }^{25}$ though these studies were of retrospective design without randomised treatment. Nevertheless it appears that for functional improvement to occur more viable myocardium is needed than for improvement in prognosis. How much viable myocardium is needed to be clinically relevant remains to be established in randomised prospective studies focusing on both functional improvement and prognosis.

\section{Perfusion alone versus perfusion and metabolism}

Detection of reduced myocardial perfusion (without any additional information about metabolism) has been proposed as a method for differentiating viable from non-viable myocardium. Thallium-201 or technetium-99m labelled tracers have been used in various studies to assess myocardial viability. ${ }^{26}$ However, it appears that the diagnostic accuracy of perfusion imaging for detecting viable tissue is less than that of perfusion imaging combined with FDG metabolic imaging. Three studies have shown that metabolic evidence of viability may be present while perfusion is absent. Sawada and colleagues studied 20 patients with a previous myocardial infarction and reported that evidence of viability obtained by FDG PET was still present in 50\% of segments which had technetium-99m activity of less than $40 \%{ }^{27}$ Soufer and associates, in a study of 37 patients with coronary artery disease, found that FDG PET accurately predicted functional improvement after revascularisation in segments that were non-viable with technetium-99m imaging but viable with FDG metabolic imaging. ${ }^{28}$ Altehoefer and colleagues studied 111 patients with coronary artery disease and showed that $5-11 \%$ of segments with technetium-99m activity of $30 \%$ or less were viable according to FDG imaging. ${ }^{29}$
Our study supports those findings. When only perfusion was used to indicate viable myocardium, 56 patients (54\%) showed significant viability, while a combination of perfusion and FDG imaging identified 63 patients $(61 \%)$ with significantly viable myocardium. Hence, of the 48 patients with no significant viability by perfusion imaging alone, seven additional patients ( $15 \%$ ) had significantly viable myocardium when the information on metabolic imaging was added. This is of clinical relevance, as about one in seven patients initially thought not to have a significant amount of viable tissue on perfusion imaging were found to be eligible for revascularisation when the combined test was used. It thus appears that perfusion imaging alone underestimates viability, and the combination of perfusion imaging and FDG metabolic imaging is a better way of discriminating between viable and non-viable myocardium. Furthermore, combined imaging has been shown to provide better discrimination between segments with a relatively high and a relatively low likelihood of recovery of function after revascularisation. ${ }^{30}$

\section{Study limitations}

The centre where this study took place is a tertiary referral centre and thus a pre-existing patient selection bias may be present.

The SPECT imaging protocol did not include administration of nitrates before tracer injection. Nitrate administration may enhance the detection of myocardial viability with To-99mtetrofosmin SPECT in patients with coronary artery disease and left ventricular dysfunction. ${ }^{31}$

During SPECT data reconstruction, no attenuation correction was applied and this may have influenced the accuracy of the results. Matsunari and colleagues reported that the use of attenuation corrected Tc-99m-tetrofosmin SPECT improved the detection of viable myocardium, mainly by decreasing the underestimation of viability in the inferior septal region. ${ }^{32}$

The findings in our study did not include the results of recovery of function after revascularisation. However, that was not a goal of the study. Our aim was specifically to quantify the presence of significant myocardial viability in patients with chronic coronary artery disease, depressed LVEF, and heart failure symptoms.

\section{Conclusions}

On the basis of the presence of viable dysfunctional myocardium, $61 \%$ of patients with chronic coronary artery disease and a depressed left ventricular ejection fraction presenting with heart failure symptoms may be considered for coronary revascularisation. The combination of perfusion and metabolic imaging identified more patients with viable myocardium than perfusion imaging alone.

\section{Authors' affiliations}

A F L Schinkel, F B Sozzi, E Boersma, A Elhendy, J R T C Roelandt, D Poldermans, Department of Cardiology, Erasmus Medical Center, Rotterdam, Netherlands

R Valkema, Nuclear Medicine, Erasmus Medical Centre

J J Bax, Department of Cardiology, Leiden University Medical Center, Leiden, Netherlands

\section{REFERENCES}

1 Bourassa MG, Gurne O, Bangdiwala SI, et al. Natural history and patterns of current practice in heart failure. The studies of left ventricular dysfunction (SOLVD) investigators. J Am Coll Cardiol 1993;22(suppl): 14-19A.

2 Hamer AW, Takayama M, Abraham M, et al. End-systolic volume and long-term survival after coronary artery bypass graft surgery in patients with impaired left ventricular function. Circulation 1994;90:2899-904.

3 Gheorghiade M, Bonow RO. Chronic heart failure in the United States a manifestation of coronary artery disease. Circulation 1998;97:282-9.

4 Ghali JK, Cooper R, Ford E. Trends in hospitalization rates for heart failure in the United States, 1973-1986: evidence for increasing population prevalence. Arch Intern Med 1990;150:769-73. 
5 Pfeffer MA, Braunwald E, Moye LA, et al, on behalf of the SAVE investigators. Effect of captopril on mortality and morbidity in patients with left ventricular dysfunction after myocardial infarction: results of the survival and ventricular enlargement trial. N Engl J Med 1992; 327:669-77.

6 Pitt B, Zannad F, Remme WJ, et al, for the randomized aldactone evaluation study investigators. The effect of spironolactone on morbidity and mortality in patients with severe heart failure. N Engl J Med 1999;341:709-17.

7 CIBIS-II Investigators. The cardiac insufficiency bisoprolol study II (CIBIS-II): a randomised trial. Lancet 1999;353:9-13.

8 Baker DW, Jones R, Hodges J, et al. Management of heart failure. III. The role of revascularization in the treatment of patients with moderate or severe left ventricular systolic dysfunction. JAMA 1994;272:1528-34.

9 Elefteriades JA, Tolis $G$, Levi $E$, et al. Coronary artery bypass grafting in severe left ventricular dysfunction: excellent survival with improved ejection fraction and functional state. J Am Coll Cardio 1993;22:1411-17.

10 Rahimtoola SH. The hibernating myocardium. Am Heart J 1989:117:211-21.

11 Diamond GA. Hibernating myocardium. Am Heart J 1989;118:1361.

12 Bax JJ, Cornel JH, Visser FC, et al. F18-fluorodesoxyglucose single-photon emission computed tomography predicts functional outcome of dyssynergic myocardium after surgical revascularization. J Nucl Cardiol 1997:4:302-8.

13 Bonow RO. Identification of viable myocardium. Circulation 1996;94:2674-80

14 Al-Mohammad A, Mahy IR, Norton MY, et al. Prevalence of hibernating myocardium in patients with severely impaired ischaemic left ventricles. Heart 1998;80:559-64.

15 Auerbach MA, Schöder H, Hoh C, et al. Prevalence of myocardial viability as detected by positron emission tomography in patients with ischemic cardiomyopathy. Circulation 1999;99:2921-6.

16 Sandler MP, Videlefsky S, Delbeke D, et al. Evaluation of myocardial ischemia using a rest metabolism/stress perfusion protocol with fluorine-1 8 deoxyglucose/technetium- $99 \mathrm{~m} \mathrm{MIBI}$ and dual-isotope simultaneous-acquisition single-photon emission computed tomography. $J$ Am Coll Cardiol 1995;26:870-80.

17 Sandler MP, Bax JJ, Patton JA, et al. Fluorine-18-fluorodeoxyglucose cardiac imaging using a modified scintillation camera. J Nucl Med 1998;39:2035-43

18 Bourdillon PD, Broderik TM, Sawada SG, et al. Regional wall motion index for infarct and non-infarct region after reperfusion in acute myocardial infarction: comparison with global wall motion index. J Am Soc Echocardiogr 1989;2:398-407.

19 Nuutila P, Knuuti J, Raitakari M, et al. Effect of antilipolysis on heart and skeletal muscle glucose uptake in overnight fasted humans. Am J Physiol 1994;267:E941-6.

20 Rambaldi R, Poldermans D, Bax JJ, et al. Dobutamine stress echocardiography and technetium-99m-tetrofosmin/fluorine 18-fluorodeoxyglucose single-photon emission computed tomography and influence of resting ejection fraction to assess myocardial viability in patients with severe left ventricular dysfunction and healed myocardial infarction. Am J Cardiol 1999;84:130-4.

21 Bax JJ, Poldermans D, Elhendy A, et al. Improvement of left ventricular ejection fraction, heart failure symptoms and prognosis after revascularization in patients with chronic coronary artery disease and viable myocardium detected by dobutamine stress echocardiography. $J$ Am Coll Cardiol 1999:34:163-9.

22 Pasquet A, Laver MS, Williams M, et al. Prediction of global left ventricular function after bypass surgery in patients with severe left ventricular dysfunction. Impact of pre-operative myocardial function, perfusion, and metabolism. Eur Heart J 2000;21:125-36.

23 Fath-Ordoubadi F, Pagano D, Marinho NVS, et al. Coronary revascularization in the treatment of moderate and severe postischemic left ventricular dysfunction. Am J Cardiol 1998;82:26-31

24 Di Carli MF, Davidson M, Little R, et al. Value of metabolic imaging with positron emission tomography for evaluating prognosis in patients with coronary artery disease and left ventricular dysfunction. Am J Cardiol 1994;73:527-33

25 Bax JJ, Wijns W. Fluorodeoxyglucose imaging to assess myocardial viability: PET, SPECT or gamma camera coincidence imaging? J Nucl Med 1999;40: 1893-5.

26 Bax JJ, Wiins W, Cornel JH, et al. Accuracy of currently available techniques for prediction of functional recovery after revascularization in patients with left ventricular dysfunction due to chronic coronary artery disease: comparison of pooled data. J Am Coll Cardiol 1997;30:1451-60.

27 Sawada SG, Allman KC, Muzik O, et al. Positron emission tomography detects evidence of viability in rest technetium-99m sestamibi defects. $J$ Am Coll Cardiol 1994;23:92-8.

28 Soufer R, Dey HM, Ng CK, et al. Comparison of sestamibi single-photon emission computed tomography with positron emission tomography for estimating left ventricular myocardial viability. Am J Cardiol 1995;75:1214-19.

29 Altehoefer C, Vom Dahl J, Biedermann M, et al. Significance of defect severity in technetium-99m-MIBI SPECT at rest to assess myocardial viability: comparison with fluorine-18-FDG PET. J Nucl Med 1994;35:569-74

30 Bax JJ, Visser FC, Elhendy A, et al. Prediction of improvement of regional left ventricular function after revascularization using different perfusion-metabolism criteria. J Nucl Med 1999;40:1866-73.

31 Flotats A, Carrio I, Estorch M, et al. Nitrate administration to enhance the detection of myocardial viability by technetium- $99 \mathrm{~m}$ tetrofosmin single-photon emission tomography. Eur J Nucl Med 1997;24:767-73.

32 Matsunari I, Boning G, Ziegler SI, et al. Attenuation-corrected $99 \mathrm{mTc}$-tetrofosmin single-photon emission computed tomography in the detection of viable myocardium: comparison with positron emission tomography using 18F-fluorodeoxyglucose. J Am Coll Cardiol 1998;32:926-35

\section{IMAGES IN CARDIOLOGY}

\section{Spontaneous right coronary artery dissection}

A 4 year old woman presented with an eight month history of recurrent exerional chest and back pain. A diagnosis of Marfan syndrome had been made in 1985; in 1988, following the discovery of a chronic type A dissection, she underwent a Starr Edwards aortic valve and ascending aortic replacement with reimplantation of the coronary arteries.

On this presentation magnetic resonance scanning showed a dissection flap extending across the aortic arch that had been present and stable for 13 years. Myocardial perfusion scanning demonstrated a reduction in tracer uptake in the inferior wall. Coronary angiography revealed a spontaneous distal right coronary artery (RCA) dissection in a tortuous vessel (panel A, black arrow). The RCA was cannulated using a 6 French Judkins right guide catheter and two guide wires Direct stenting of the lesion was performed uneventfully with a $3.5 \times 23$ Sonic stent (Cordis) (panel B, white arrow). Following the procedure the patient remains well.

\section{S R Baker}

C Knight

A Deaner
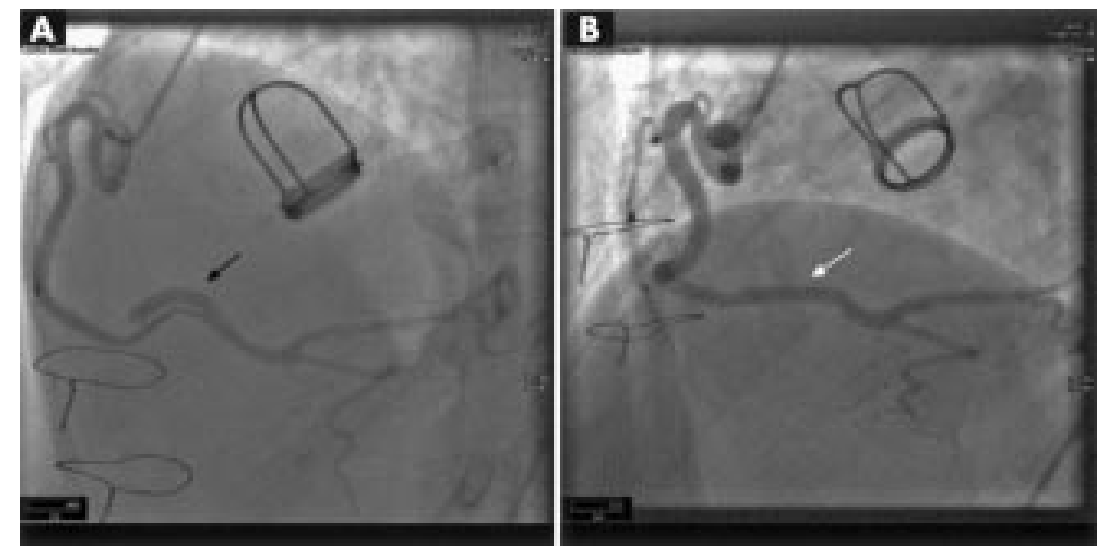